\title{
Type 1 and type 2 diabetes after gestational diabetes: a 23 year cohort study
}

\author{
Anna-Maaria Auvinen ${ }^{1,2} \cdot$ Kaisu Luiro $^{3}$ (D) Jari Jokelainen ${ }^{4,5}$ (D) Ilkka Järvelä ${ }^{6}$ Mikael Knip ${ }^{7,8,9}$ (D) Juha Auvinen ${ }^{4,5}$. \\ Juha S. Tapanainen ${ }^{1,2,3}$ (D)
}

Received: 18 November 2019 / Accepted: 11 May 2020 / Published online: 29 July 2020

(C) The Author(s) 2020

\begin{abstract}
Aims/hypothesis The aim of this work was to examine the progression to type 1 and type 2 diabetes after gestational diabetes mellitus (GDM) in a 23 year follow-up study.

Methods We carried out a cohort study of 391 women with GDM diagnosed by an OGTT or the use of insulin treatment during pregnancy, and 391 age- and parity-matched control participants, who delivered in 1984-1994 at the Oulu University Hospital, Finland. Diagnostic cut-off levels for glucose were as follows: fasting, $\geq 4.8 \mathrm{mmol} / \mathrm{l} ; 1 \mathrm{~h}, \geq 10.0 \mathrm{mmol} / \mathrm{l} ;$ and $2 \mathrm{~h}, \geq 8.7 \mathrm{mmol} / \mathrm{l}$. Two follow-up questionnaires were sent (in 1995-1996 and 2012-2013) to assess the progression to type 1 and type 2 diabetes. Mean follow-up time was 23.1 (range 18.7-28.8) years.

Results Type 1 diabetes developed (5.7\%) during the first 7 years after GDM pregnancy and was predictable at a $2 \mathrm{~h}$ OGTT value of $11.9 \mathrm{mmol} / \mathrm{l}$ during pregnancy (receiver operating characteristic analysis: AUC 0.91 , sensitivity $76.5 \%$, specificity $96.0 \%$ ). Type 2 diabetes increased linearly to $50.4 \%$ by the end of the follow-up period and was moderately predictable with fasting glucose (AUC 0.69, sensitivity $63.5 \%$, specificity $68.2 \%$ ) at a level of $5.1 \mathrm{mmol} / \mathrm{l}$ (identical to the fasting glucose cut-off recommended by the International Association of Diabetes and Pregnancy Study Groups [IADPSG) and WHO]).

Conclusions/interpretation All women with GDM should be intensively monitored for a decade, after which the risk for type 1 diabetes is minimal. However, the incidence of type 2 diabetes remains linear, and therefore individualised lifelong follow-up is recommended.
\end{abstract}

Keywords GDM · Insulin · OGTT · Prediction · Type 1 diabetes · Type 2 diabetes

Anna-Maaria Auvinen and Kaisu Luiro contributed equally to this work.

Electronic supplementary material The online version of this article (https://doi.org/10.1007/s00125-020-05215-3) contains peer-reviewed but unedited supplementary material, which is available to authorised users.

Juha S. Tapanainen

juha.tapanainen@helsinki.fi

1 Department of Obstetrics and Gynecology, Oulu University Hospital, Oulu, Finland

2 PEDEGO Research Unit, Medical Research Centre, University of Oulu, Oulu, Finland

3 Department of Obstetrics and Gynecology, University of Helsinki and Helsinki University Hospital, PO 140 (Haartmaninkatu 2E), 00029 Helsinki, Finland

4 Center for Life Course Health Research, University of Oulu, Oulu, Finland
5 Unit of Primary Care, Oulu University Hospital, Oulu, Finland

6 Department of Obstetrics and Gynecology, Kuopio University Hospital, Kuopio, Finland

7 Children's Hospital, University of Helsinki and Helsinki University Hospital, Helsinki, Finland

8 Research Program for Clinical and Molecular Metabolism, Faculty of Medicine, University of Helsinki, Helsinki, Finland

9 Tampere Center for Child Health Research, Tampere University Hospital, Tampere, Finland 


\section{Research in context}

\section{What is already known about this subject?}

- The reported incidence of type 2 diabetes mellitus after gestational diabetes mellitus (GDM) varies from $3 \%$ to $70 \%$, while the incidence of type 1 diabetes is $5-7 \%$ in Europe

- In published studies the follow-up time has varied from 6 months to 15 years

What is the key question?

- What is the long-term progression to diabetes after GDM, and how long should these women be monitored?

What are the new findings?

- Type 1 diabetes developed in $5.7 \%$ of women and all were diagnosed within 7 years after the GDM pregnancy

- Of women with GDM, 50.4\% developed type 2 diabetes and the incidence remained linear until the end of the follow-up of 23.1 years

- Fasting glucose values above $5.1 \mathrm{mmol} / \mathrm{l}$ were predictive of future type 2 diabetes development

How might this impact on clinical practice in the foreseeable future?

- Women with high glucose values in OGTT and requiring insulin treatment during pregnancy should be carefully monitored for a decade for type 1 diabetes; due to the linear increase in type 2 diabetes during the entire followup period, regular and individualised lifelong monitoring is recommended after GDM

\begin{abstract}
Abbreviations
GDM Gestational diabetes mellitus

ROC Receiver operating characteristic
\end{abstract}

\section{Introduction}

The prevalence of gestational diabetes mellitus (GDM) [1] and type 2 diabetes [2] is increasing worldwide and studies have shown that women with GDM are at high risk of developing diabetes later in life. However, the reported incidence of type 2 diabetes after GDM varies considerably from $3 \%$ to $70 \%$ depending on the follow-up time and ethnicity [3-5]. The overall incidence of type 1 diabetes mellitus after GDM is significantly lower, at 5-7\% in Europe, but the risk increases with the number of positive autoantibodies and the duration of follow-up after pregnancy [6].

Insulin resistance increases during pregnancy because of increasing weight and adiposity combined with reduced insulin sensitivity by placental hormones. In relatively short follow-up studies (up to 15 years), high glucose levels in an OGTT, age, BMI, insulin treatment during pregnancy and a single autoantibody positivity have been predictive factors of later onset of diabetes [7-9].

We previously reported a prospective 6 year follow-up study of women with GDM and healthy control counterparts, showing that $4.6 \%$ of the GDM cohort developed type 1 diabetes and $5.3 \%$ developed type 2 diabetes, while none of the control group became diabetic [6]. We report here the results of the 23 year follow-up study of these women regarding the progression to type 1 and type 2 diabetes after GDM, including demographic, diagnostic and treatment data.

\section{Methods}

Study population and design The population and design of this study has been described previously [6], and further information can be found in the electronic supplementary material (ESM) Methods. Briefly, this cohort study included 435 white women with GDM and a singleton pregnancy. The control cohort of 435 white women was pair-matched by age ( \pm 2 years), parity and date of delivery ( \pm 2 days). Both cohorts delivered at the Oulu University Hospital, Finland. Only women with GDM diagnosed by oral glucose tolerance test (OGTT) $(n=363)$ or who were treated with insulin $(n=28)$, and their matched control counterparts $(n=391)$, were included in further analysis. The cut-off values for the glucose concentrations were set according to the recommendation of the Finnish Diabetes Association: fasting, $\geq 4.8 \mathrm{mmol} / \mathrm{l} ; 1 \mathrm{~h}$, $\geq 10.0 \mathrm{mmol} / \mathrm{l}$; and $2 \mathrm{~h}, \geq 8.7 \mathrm{mmol} / \mathrm{l}$. Any single abnormal value in the OGTT was considered diagnostic for GDM.

Questionnaire-based follow-up An invitation to participate in this study was sent in 1995-1996 (1-11 years after pregnancy) along with the first follow-up questionnaire and an informed consent form. The questionnaire included questions about GDM treatment (diet or insulin), pre-pregnancy weight and height, progression to diabetes, time of diagnosis and diabetes medication. 
A second questionnaire was sent out in 2012-2013. Thirteen women with GDM (3.3\%) and six control participants (1.5\%) had died. Finally, 297 women with GDM and 297 control participants $(76.0 \%)$ took part in the study. The mean follow-up period from delivery to the date of completing the questionnaire was 23.1 (range 18.7-28.8) years in the GDM cohort and 23.3 (range 18.9-30.1) years in the control cohort.

Statistical analysis Comparisons of baseline demographic characteristics between the groups were performed using one-way ANOVA. Kaplan-Meier survival curves were used to compare the development of type 1 or type 2 diabetes after pregnancy. To find the best predictive model for progression to type 1 and type 2 diabetes, sensitivity and specificity, receiver operating characteristic (ROC) curves were constructed with continuous glucose values. AUC was used in the classification analysis. The analyses were performed with IBM SPSS Statistics for Windows (versions 21 and 25, IBM, Armonk, NY) and RStudio (Boston, MA) software. The figures were produced using the ggplot2 ( $\mathrm{R}$ package version 0.4.6., https://CRAN.R-project.org/package=survminer) and Adobe Illustrator (Adobe Systems, San Jose, CA).

\section{Results}

At the end of the follow-up period, the mean $\pm \mathrm{SD}$ age of the GDM cohort was $54.7 \pm 6.4$ years, and that of the control cohort was $55.3 \pm 6.4$ years. Body weight and BMI were higher in the GDM group than the control group, as expected (ESM Tables 1,2). However, weight gain and increase in BMI during pregnancy was higher in the control group. The influence of age on disease progression is depicted in ESM Fig. 1 and in ESM Results.

During the follow-up study, $53.2 \%$ of women in the GDM group developed diabetes (type 1, 5.7\%; type 2, 50.4\%) and $5.5 \%$ developed type 2 diabetes in the control group (Fig. 1). No-one in the control group developed type 1 diabetes. Incidence of type 2 diabetes increased linearly until the end of the study, while all the participants who developed type 1 diabetes were diagnosed during the first 7 years after pregnancy. Details of the OGTT measurements are shown in ESM Table 3.

\section{OGTT glucose levels in prediction of disease progression} Pathological glucose value at any of the three OGTT time points was associated with a shorter time to progression to type 1 or type 2 diabetes (ESM Fig. 2). Moreover, three abnormal glucose values in OGTT were most predictive of both type 1 and type 2 diabetes occurrence (Fig. 2). Altogether, $60.9 \%$ of the women with abnormal fasting glucose developed type 2 diabetes. In the ROC analyses with continuous glucose values, the most predictive single OGTT value regarding type 1 diabetes was the $2 \mathrm{~h}$ glucose value (AUC 0.91 , sensitivity $76.5 \%$, specificity $96.0 \%$ at a glucose level of $11.9 \mathrm{mmol} / \mathrm{l}$ ). The most predictive OGTT value for type 2 diabetes was fasting glucose (AUC 0.69 , sensitivity $63.5 \%$, specificity $68.2 \%$ at a glucose level of $5.1 \mathrm{mmol} / \mathrm{l}$ ) (ESM Table 4).

To dissect undiagnosed diabetes in our GDM cohort, we analysed a subgroup 'diabetes in pregnancy' comprising of women with fasting glucose $\geq 7.0 \mathrm{mmol} / \mathrm{l}$ or $2 \mathrm{~h}$ glucose $\geq 11.1 \mathrm{mmol} / \mathrm{l}$, according to the WHO criteria [10]. Fortyeight $(12.3 \%)$ women belonged to this subgroup based on their OGTT. During the follow-up period, 13 (27\%) women in this subgroup developed type 1 diabetes and $22(46 \%)$ women developed type 2 diabetes.

Insulin treatment for GDM in prediction of disease progression Women who received insulin treatment for GDM had a higher probability of subsequent diagnosis of both type 1 and type 2 diabetes (ESM Fig. 3). Furthermore, the time to diabetes diagnosis was longer in women without insulin treatment for GDM. Conversely, only a few women (1.2\%) without insulin treatment developed type 1 diabetes. The sensitivity of insulin treatment to predict type 1 diabetes was $90.5 \%$ and specificity $64.7 \%$ (AUC 0.78 ) according to the ROC analyses; sensitivity as regards predicting type 2 diabetes was $56.9 \%$, and specificity $74.6 \%$ (AUC 0.66) (ESM Table 4).

\section{Discussion}

This 23 year prospective cohort study demonstrated that after GDM, $5.7 \%$ of women developed type 1 diabetes and approximately $50 \%$ developed type 2 diabetes. Type 1 diabetes was diagnosed less than a decade after the GDM, while the incidence for type 2 diabetes remained linear until the end of the study.

To our knowledge, this is one of the longest prospective follow-up studies on women with GDM. The majority of the diagnoses $(92.8 \%)$ were made based on OGTT, albeit using capillary glucose determinations. In addition, the participation rate $(76 \%)$ was remarkably high considering the long followup period. The main weakness of the study is the self-reported disease progression. Had OGTT been performed systematically as part of the follow-up, the incidence of type 2 diabetes might have been higher in both cohorts, as the prevalence of undiagnosed disease is reported to be as high as $20 \%$ in the general population [11]. It is very unlikely that the participants would have mixed up the type of diabetes that they have, as the diagnosis, treatment and follow-up of type 1 diabetes takes place in a specialised hospital setting while type 2 diabetes care occurs at the health-centre level. However, it is worth noting that the incidence of type 1 diabetes among young adults is higher in 
a

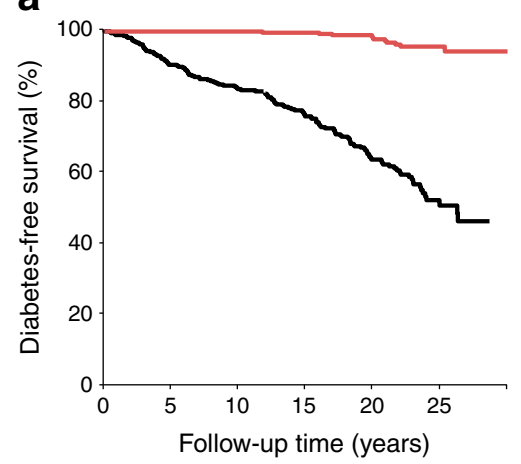

$N$ at risk

$\begin{array}{llllllll}\text { Control cohort } & 391 & 356 & 313 & 299 & 257 & 90 & 1\end{array}$ b

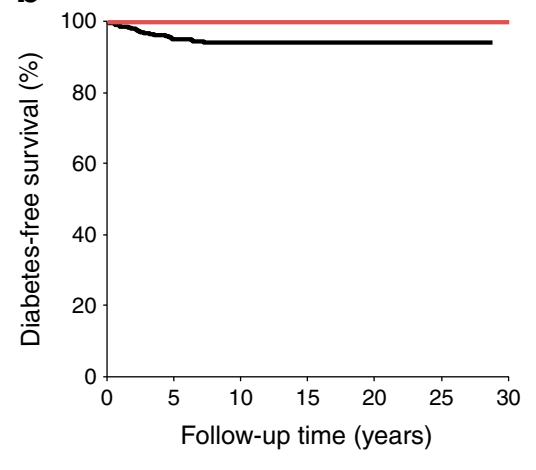

\begin{tabular}{ccccccc}
391 & 356 & 313 & 299 & 257 & 90 & 1 \\
391 & 326 & 263 & 228 & 164 & 37 & 0 \\
\cline { 3 - 5 } & \multicolumn{1}{c}{ Control cohort } & \multicolumn{3}{c}{ GDM cohort }
\end{tabular}
C

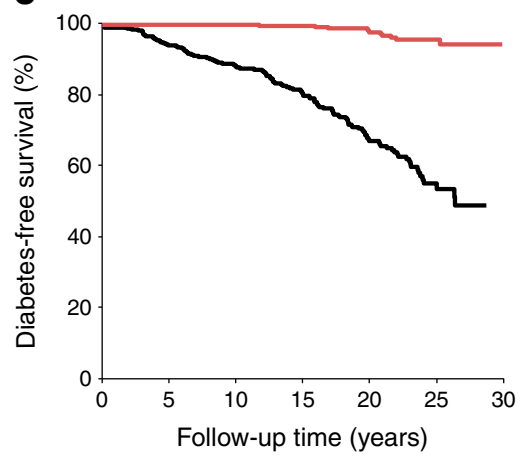

$\begin{array}{lllllll}391 & 356 & 313 & 299 & 257 & 90 & 1 \\ 391 & 326 & 263 & 228 & 164 & 37 & 0\end{array}$
Fig. 1 Probability of remaining free from (a) diabetes, (b) type 1 diabetes or (c) type 2 diabetes among women with and without GDM. Logrank $p<0.001$ in all figure parts. Mean $(95 \% \mathrm{CI})$ diabetes-free survival time in women with vs without GDM was as follows: diabetes, $21.5(20.5,22.4)$

Finland than in other countries [12]. At the time of this study, a risk-based screening strategy for GDM was used in Finland, while nowadays nearly universal screening is recommended, and this may have underestimated the incidence of GDM.

Few prior studies compared the predictive value of single glucose levels or their combinations in OGTT regarding type 2 diabetes development. In the current study, $60.9 \%$ of the women with abnormal fasting glucose developed type 2 diabetes. Consistent with our results, three other studies reported that abnormal fasting glucose is the strongest years vs $29.6(29.3,29.9)$ years; type 1 diabetes, $26.7(25.8,27.5)$ years vs no occurrence of type 1 diabetes; and type 2 diabetes, $22.6(21.7,23.5)$ years vs $29.6(29.3,29.9)$ years

predictor of later type 2 diabetes [13]. Conflicting results have also been reported, showing abnormal $1 \mathrm{~h}$ glucose or $2 \mathrm{~h}$ glucose to be the strongest predictor of type 2 diabetes [14]. However, the glucose load in OGTT also varied from $50 \mathrm{~g}$ to $75 \mathrm{~g}$ or $100 \mathrm{~g}$ among studies. In our study, each OGTT time point was independently predictive of type 2 diabetes, justifying the inclusion of the $1 \mathrm{~h}$ test, which is omitted in some countries. Remarkably, the identical cut-off for fasting glucose $(5.1 \mathrm{mmol} / \mathrm{l})$ that was associated with the future type 2 diabetes risk in this study, was associated with offspring risk and a

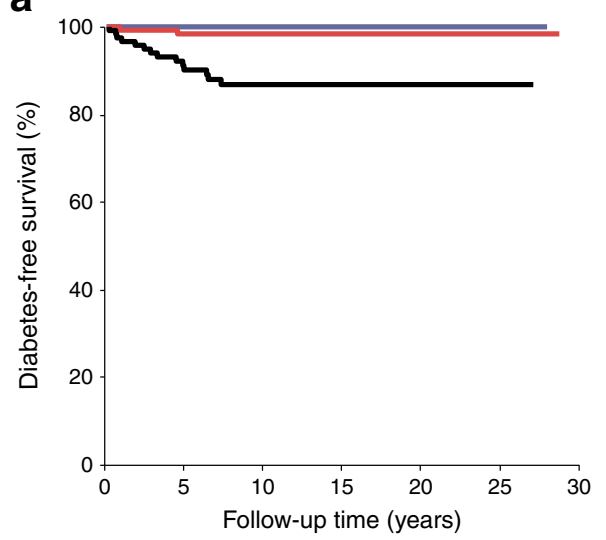

b

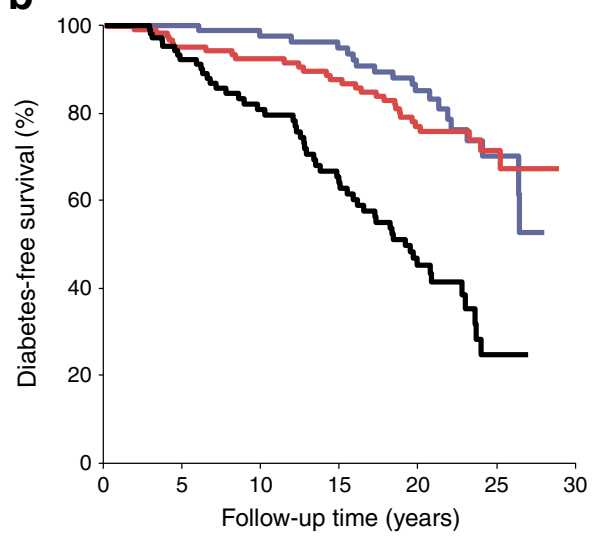

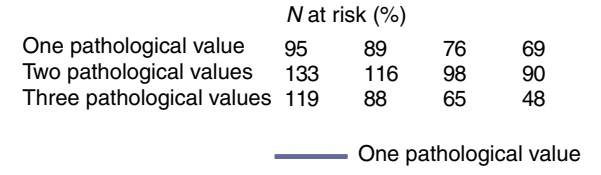

One pathological value

Fig. 2 Probability of remaining free from (a) type 1 diabetes and (b) type 2 diabetes according to the number of pathological glucose values in OGTT during the GDM pregnancy. Logrank $p<0.001$. Mean $(95 \% \mathrm{CI})$ type 1 diabetes-free survival time was as follows: one pathological value, no occurrence of type 1 diabetes; two pathological values, 28.2 (27.5,

\begin{tabular}{|c|c|c|c|c|c|c|}
\hline \multicolumn{7}{|c|}{$N$ at risk (\%) } \\
\hline 95 & 89 & 76 & 69 & 54 & 13 & 0 \\
\hline 133 & 116 & 98 & 90 & 66 & 18 & 0 \\
\hline 119 & 88 & 65 & 48 & 28 & 4 & 0 \\
\hline
\end{tabular}

29.0) years; three pathological values, 21.4 (18.8, 24.0) years. Mean $(95 \% \mathrm{CI})$ type 2 diabetes-free survival time was as follows: one pathological value, $25.0(23.8,26.1)$ years; two pathological values, 24.3 (22.9, $25.8)$ years; three pathological values, $17.9(16.2,19.6)$ years 
maternal complications in the Hyperglycemia and Adverse Pregnancy Outcomes (HAPO) study, from which the current International Association of Diabetes and Pregnancy Study Groups (IADPSG)/WHO diagnostic guidelines are derived $[10,15,16]$. Therefore, the present study further validates the use of the lower fasting glucose cut-offs for GDM diagnosis.

When examining the predictive value of OGTT glucose levels in terms of progression to type 1 diabetes, the most predictive single OGTT time point for later type 1 diabetes was the $2 \mathrm{~h}$ glucose concentration with a cut-off of $11.9 \mathrm{mmol} / \mathrm{l}$, which is higher than the current diagnostic level for GDM (7.8-8.6 mmol/l). In addition, most of the women $(72.9 \%)$ who fulfilled the WHO criteria for the 'diabetes in pregnancy' subgroup were later diagnosed with diabetes.

In the present study, insulin treatment during pregnancy was a strong predictor for both type 1 and type 2 diabetes, which is in agreement with several earlier studies $[5,6,12$, 13]. Moreover, women who had received insulin treatment for GDM developed diabetes earlier, and only a few women $(1.2 \%)$ without insulin treatment developed type 1 diabetes. During the first 10 years of follow-up, the development of type 2 diabetes was rare in women without insulin treatment for GDM, but thereafter the disease progression curve was almost linear and parallel to that of women on insulin for GDM. This reflects the association of exogenous insulin treatment with the severity of an impaired glucose tolerance, also predictive of higher diabetes progression rate later in life. In addition, being over 30 years of age at the time of GDM increased the progression rate to type 2 diabetes. However, this association disappeared when the glucose levels from the OGTT were included in the same model, which suggests that abnormal glucose concentrations may be more important indicators of later type 2 diabetes.

In conclusion, women with GDM, especially those on insulin treatment, should be carefully monitored for the first decade after the pregnancy, after which the risk for type 1 diabetes becomes negligible. However, the risk for type 2 diabetes remains and warrants an individualised, lifelong follow-up.

Acknowledgements We thank K. Teramo (University of Helsinki) for advice and valuable comments during the study.

Data availability The original data are available on request from the corresponding author.

Funding Open access funding provided by the University of Helsinki including Helsinki University Central Hospital. This study was supported by Sigrid Jusélius Foundation, Academy of Finland, and Helsinki and Oulu University Hospital Research Funds.

Authors' relationships and activities The authors declare that there are no relationships or activities that might bias, or be perceived to bias, their work.
Contribution statement JST and AMA designed the study. JST obtained funding for the study and supervised the project. All authors contributed to data collection and data analyses. AMA, JA and JJ performed the statistical analyses. AMA wrote the first draft of the manuscript; all authors contributed to revision and approved the final version of the manuscript. JST is the guarantor of this work.

Open Access This article is licensed under a Creative Commons Attribution 4.0 International License, which permits use, sharing, adaptation, distribution and reproduction in any medium or format, as long as you give appropriate credit to the original author(s) and the source, provide a link to the Creative Commons licence, and indicate if changes were made. The images or other third party material in this article are included in the article's Creative Commons licence, unless indicated otherwise in a credit line to the material. If material is not included in the article's Creative Commons licence and your intended use is not permitted by statutory regulation or exceeds the permitted use, you will need to obtain permission directly from the copyright holder. To view a copy of this licence, visit http://creativecommons.org/licenses/by/4.0/.

\section{References}

1. Ferrara A (2007) Increasing prevalence of gestational diabetes mellitus: a public health perspective. Diabetes Care 30(Suppl 2): 141-146. https://doi.org/10.2337/dc07-s206

2. Chen L, Magliano DJ, Zimmet PZ (2012) The worldwide epidemiology of type 2 diabetes mellitus: present and future perspectives. Nat Rev Endocrinol 8:228-236. https://doi.org/10.1038/nrendo. 2011.183

3. O'Sullivan JB (1989) The Boston Gestational Diabetes Studies: review and perspectives. In: Sutherland HW, Stowers JM, Pearson DWM (eds) Carbohydrate metabolism in pregnancy and the new-born, vol 4. Springer-Verlag, London, pp 287-294

4. Bellamy L, Casas JP, Hingorani AD, Williams D (2009) Type 2 diabetes mellitus after gestational diabetes: a systematic review and meta-analysis. Lancet 373:1773-1779. https://doi.org/10.1016/ S0140-6736(09)60731-5

5. Chamberlain CR, Oldenburg B, Wilson AN et al (2016) Type 2 diabetes after gestational diabetes: greater than fourfold risk among Indigenous compared with non-Indigenous Australian women. Diabetes Metab Res Rev 32:217-227. https://doi.org/10.1002/ dmrr.2715

6. Järvelä IY, Juutinen J, Koskela P et al (2006) Gestational diabetes identifies women at risk for permanent type 1 and type 2 diabetes in fertile age: predictive role of autoantibodies. Diabetes Care 29:607612. https://doi.org/10.2337/diacare.29.03.06.dc05-1118

7. Göbl CS, Bozkurt L, Prikoszovich T, Winzer C, Pacini G, KautzkyWiller A (2011) Early possible risk factors for overt diabetes after gestational diabetes mellitus. Obstet Gynecol 118:71-78. https:// doi.org/10.1097/AOG.0b013e318220e18f

8. Chew WF, Rokiah P, Chan SP, Chee WS, Lee LF, Chan YM (2012) Prevalence of glucose intolerance and associated antenatal and historical risk factors among Malaysian women with a history of gestational diabetes mellitus. Singapore Med J 53:814-820

9. Papadopoulou A, Lynch KF, Anderberg E et al (2012) HLA-DQB1 genotypes and islet cell autoantibodies against GAD65 and IA-2 in relation to development of diabetes post partum in women with gestational diabetes mellitus. Diabetes Res Clin Pract 95:260 264. https://doi.org/10.1016/j.diabres.2011.10.037

10. World Health Organization (2014) Diagnostic criteria and classification of hyperglycaemia first detected in pregnancy: a World Health Organization guideline. Diabetes Res Clin Pract 103:341363. https://doi.org/10.1016/j.diabres.2013.10.012 
11. Heltberg A, Andersen JS, Sandholdt H, Siersma V, Kragstrup J, Ellervik C (2018) Predictors of undiagnosed prevalent type 2 diabetes - The Danish General Suburban Population Study. Prim Care Diabetes 12:13-22. https://doi.org/10.1016/j.pcd.2017.08.005

12. Lammi N, Taskinen O, Moltchanova E et al (2007) A high incidence of type 1 diabetes and an alarming increase in the incidence of type 2 diabetes among young adults in Finland between 1992 and 1996. Diabetologia 50:1393-1400. https://doi.org/10.1007/s00125007-0690-4

13. Eades CE, Styles M, Leese GP, Cheyne H, Evans JM (2015) Progression from gestational diabetes to type 2 diabetes in one region of Scotland: an observational follow-up study. BMC Pregnancy Childbirth 15:11. https://doi.org/10.1186/s12884-0150457-8
14. Dalfrà MG, Lapolla $\mathrm{A}$, Masin $\mathrm{M}$ et al (2001) Antepartum and early postpartum predictors of type 2 diabetes development in women with gestational diabetes mellitus. Diabetes Metab 27:675-680

15. The HAPO Study Cooperative Research Group (2008) Hyperglycemia and adverse pregnancy outcomes. N Engl J Med 358(19):1991-2001. https://doi.org/10.1056/NEJMoa0707943

16. International Association of Diabetes and Pregnancy Study Groups Consensus Panel, Metzger BE, Gabbe SG et al (2010) International Association of Diabetes and Pregnancy Study Groups recommendations on the diagnosis and classification of hyperglycemia in pregnancy. Diabetes Care 33:676-682. https://doi.org/10.2337/ dc09-1848

Publisher's note Springer Nature remains neutral with regard to jurisdictional claims in published maps and institutional affiliations. 